\title{
GENERALIZED DIFFUSION THEORY OF NON-ROTATIONAL, RIGID SPHERICAL PARTICLE SEDIMENTATION IN VISCOUS FLUID
}

\author{
Nguyen Hong Phan, Nguyen Van DieP \\ Institute of Mechanics, NCNST, 264 Doican, Hanoi, Vietnam
}

\begin{abstract}
This paper is devoted to application of Generalized Diffusion Theory for solving a sedimentation problem of rigid spherical particles in viscous fluid. The governing equations have been obtained. It is shown that, in this case the governing equation system is a hyperbolic one, and the equations in the characteristic form have been derived. The mathemetical properties of the obtained equation system and the solution for stationary sedimentation where investigated numericaly.
\end{abstract}

\section{The General Motion Equation System of Two-Phase Particules-} Fluid Flows

In [1-3] the Generalized Diffusion Theory of Multiphase Flows of fluids with microstructural particles (microdeformation, rotation, and arbitrary shape) has been developed. In the simplest case, the movement of viscous fluid carrying non-rotational, rigid spherical particles without external forces, except the force of gravity, is described by the following general equations system:

$$
\begin{aligned}
& \bar{\nabla} \cdot \bar{U}=0 \\
& \frac{d \varphi}{d t}=-\frac{1}{\rho_{1}} \bar{\nabla} \cdot \bar{J}, \quad \bar{J}=\rho_{1} \varphi\left(\bar{U}_{1}-\bar{U}\right) \\
& \rho \frac{d \bar{U}}{d t}=\rho \bar{g}-\bar{\nabla} P+\bar{\nabla} \cdot \bar{\tau}-\frac{\rho_{1}-\rho_{2}}{\rho_{1}} \frac{D \bar{J}}{D t} \\
& \frac{D \bar{J}}{\bar{D} t}=-\frac{T}{\alpha_{11}} \frac{\rho_{1}^{2} \varphi(1-\varphi)}{\rho_{1}+\varphi\left(\rho_{2}-\rho_{1}\right)}\left\{\bar{J}+\left[\alpha_{1}-\alpha_{11}\left(h_{1}-\frac{\rho_{2}}{\rho_{1}} h_{2}\right)\right] \frac{\bar{\nabla} T}{T^{2}} \ldots\right. \\
&\left.-\frac{1}{T} \alpha_{11}\left[\left(1-\frac{\rho_{2}}{\rho_{1}}\right) \bar{g}-\left(1-\frac{\rho_{2}}{\rho_{1}}\right) \frac{d \bar{U}}{d t}-\frac{1}{1-\varphi}\left(\bar{\nabla} \mu_{1}\right)_{P, T}\right]\right\} \\
& \rho=\rho_{1} \varphi+\rho_{2}(1-\varphi), \quad(1.1) \\
& \bar{\tau}=\frac{2 \mu}{T} \bar{e}, \quad \mu_{1}=\frac{\partial \varepsilon}{\partial c_{1}} \quad \bar{e}=\frac{1}{2}\left(\bar{\nabla} \bar{U}+\bar{\nabla} \bar{U}^{T}\right), \\
& \frac{D}{D t}(\ldots)=\frac{d}{d t}(\ldots)+[(\ldots) \cdot \bar{\nabla}] \bar{U}+(\ldots)(\bar{\nabla} \cdot \bar{U}), \quad \frac{d}{d t}(\ldots)=\frac{\partial}{\partial t}(\ldots)+(\bar{U} \cdot \bar{\nabla})(\ldots),
\end{aligned}
$$


where: $\bar{U}_{1}, \bar{U}$ are mean particles velocity and mean volume velocity of suspension; $\rho_{1}, \rho_{2} ; \rho$-densities of particles, fluid phase and suspension; $\bar{J}$ - particles generalized diffusion flux; $\varphi$ - particles volume concentration; $P$ - thermodynamical pressure; $T$ - temperature of suspension; $\bar{\tau}$ - viscous stress tensor; $\bar{e}$ - deformation velocity tensor; $h_{1}, h_{2}$ - enthalpi of solid and fluid phases; $\mu_{1}$ - generalized chemical potential of particles, $\varepsilon$ - internal energy of suspension, $c_{1}$ - particles mass concentration; $\alpha_{i}$, $\beta_{i}$ - constitutive coefficients; $g$ - acceleration of gravity; $t$ - time;

\section{The Governing Equation System of Particles Sedimentation and its} Mathematical Properties

Let's consider sedimentational process of rigid, spherical, non-rotational particles in a viscous suspension filled up the space between two horizontal parallel planes. It is assumed that the suspension is in a stationary situation and being forced by the gravity only.

In this case the movement of particles under the gravity force is considered in the upwards vertical $z$ direction only, and there is no the mean volume suspension velocity. Therefore $\varphi, J, p$ are functions of variables $(t, z)$ :

$$
\varphi=\varphi(t, z) ; \quad \bar{J}=(0,0, J) ; \quad J=J(t, z) ; \quad p=p(t, z) .
$$

On basis of the above mentioned assumptions, the equation system takes the following form:

$$
\begin{aligned}
\frac{\partial \varphi}{\partial t} & =-\frac{1}{\rho_{1}} \frac{\partial J}{\partial z} \\
\frac{\partial J}{\partial t} & =-\frac{T}{\alpha_{11}} \frac{\rho_{1}^{2} \varphi(1-\varphi)}{\rho_{1}+\varphi\left(\rho_{2}-\rho_{1}\right)} J-\frac{\rho_{1}\left(\rho_{1}-\rho_{2}\right) \varphi(1-\varphi)}{\rho_{1}+\varphi\left(\rho_{2}-\rho_{1}\right)} g-\frac{\rho_{1} D}{\rho_{1}+\varphi\left(\rho_{2}-\rho_{1}\right)} \frac{\partial \varphi}{\partial z} \\
0 & =-\frac{\partial p}{\partial z}-\rho g-\frac{\rho_{1}-\rho_{2}}{\rho_{1}} \frac{\partial J}{\partial t}
\end{aligned}
$$

In addition, it is supposed that:

$$
D=\left(\frac{\partial \mu_{1}}{\partial \varphi}\right)_{P, T} \varphi \rho_{1}=\text { const } ; \quad K_{1}=-\frac{T \rho_{1}}{\alpha_{11}}=\text { const. }
$$

In order to determine the volume concentration $\varphi$ and diffusion flux $\bar{J}$ of particles, the following conditions are used:

- Initial conditions:

$$
t=0, \quad \varphi=\varphi_{0}=\text { constant } ; \quad 0<\varphi<1 ; \quad J=J_{0}=0
$$

- Boundary conditions:

$$
z=0, L ; \quad J=0,
$$


where $L$ is a distance between the parallel planes

\subsection{The hyperbolic property of the governing equation system}

- The equation (1.1) and (2.1) can be rewritten in the form

$$
\begin{aligned}
& \frac{\partial \varphi}{\partial t}+\frac{1}{\rho_{1}} \frac{\partial J}{\partial z}=0 \\
& \frac{\partial J}{\partial t}+\frac{\rho_{1} D}{\rho_{1}+\varphi\left(\rho_{2}-\rho_{1}\right)} \frac{\partial \varphi}{\partial z}=-\frac{T}{\alpha_{11}} \frac{\rho_{1}^{2} \varphi(1-\varphi)}{\rho_{1}+\varphi\left(\rho_{2}-\rho_{1}\right)} J-\frac{\rho_{1}\left(\rho_{1}-\rho_{2}\right) \varphi(1-\varphi)}{\rho_{1}+\varphi\left(\rho_{2}-\rho_{1}\right)} g .
\end{aligned}
$$

Since $\varphi$ and $J$ are assumed derivativeable functions of $t$ and $z$ variables so:

$$
\begin{aligned}
& d \varphi=\frac{\partial \varphi}{\partial t} d t+\frac{\partial \varphi}{\partial z} d z \\
& d J=\frac{\partial J}{\partial t} d t+\frac{\partial J}{\partial z} d z
\end{aligned}
$$

Combining (2.3) and (2.4) yields the following system:

$$
\left(\begin{array}{cccc}
1 & 0 & 0 & a_{1} \\
0 & a_{2} & 1 & 0 \\
d t & d z & 0 & 0 \\
0 & 0 & d t & d z
\end{array}\right)\left(\begin{array}{c}
\varphi_{t} \\
\varphi_{z} \\
J_{t} \\
J_{z}
\end{array}\right)=\left(\begin{array}{c}
0 \\
f \\
d \varphi \\
d J
\end{array}\right)
$$

where

$$
\begin{aligned}
a_{1} & =\frac{1}{\rho_{1}} ; \quad a_{2}=\frac{D K_{\rho}}{K_{\rho}+\varphi\left(1-K_{\rho}\right)} ; \quad K_{\rho}=\frac{\rho_{1}}{\rho_{2}} ; \\
f & =K_{2} \frac{K_{\rho} \varphi(1-\varphi)}{K_{\rho}+\varphi\left(1-K_{\rho}\right)} J+K_{3} \frac{K_{\rho} \varphi(1-\varphi)}{K_{\rho}+\varphi\left(1-K_{\rho}\right)} ; \\
K_{2} & =-\frac{T}{\alpha_{11}} \rho_{1}=\text { const; } \quad K_{3}=g\left(\rho_{2}-\rho_{1}\right) .
\end{aligned}
$$

It is obvious that the eigenvalues of determinant of the matrix coefficients of the system (2.5) are:

$$
\left(\frac{d z}{d t}\right)^{2}-a_{1} a_{2}=0 \quad \text { or } \quad \frac{d z}{d t}=\lambda_{1,2}= \pm \sqrt{\frac{D}{\rho_{2}\left[K_{\rho}+\varphi\left(1-K_{\rho}\right)\right]}} .
$$

And they are equations of characteristic curves $C_{1,2}$ of the system (2.5). Due to

$$
\frac{D}{\rho_{2}\left[K_{\rho}+\varphi\left(1-K_{\rho}\right)\right]} \geq 0
$$

the system (2.5) is hyperbolic. 


\subsection{The variation of characteristic directions}

In figures $1-4$ the variation of characteristic directions depending on coefficient $D$, density ratio $K_{\rho}=\rho_{1} / \rho_{2}$ and concentration $\varphi$ is presented. In Fig. 1 it can be shown that the characteristic direction for $K_{\rho}=0.8$ (particle is lighter than fluid) ${ }^{\circ}$ is located upper the one for $K_{\rho}=1.3$ (particle is heavier than fluid).

In increasing $K_{\rho}$, the characteristic curve with bigger $D$ is located over the one with smaller $D$. This can be seen in Fig. 2.

The Figures 3, 4 demonstrate the dependence of characteristic direction on volume concentration in two cases of the density ratio: particle is lighter, and particle is heavier than fluid.

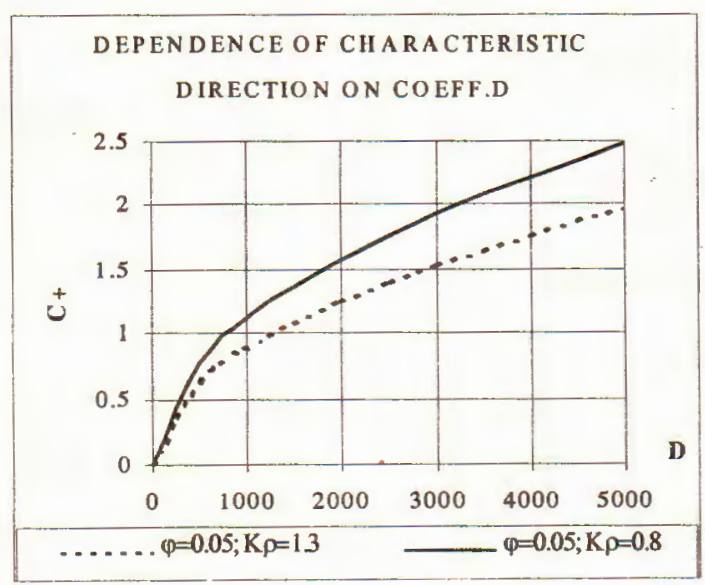

Fig. 1

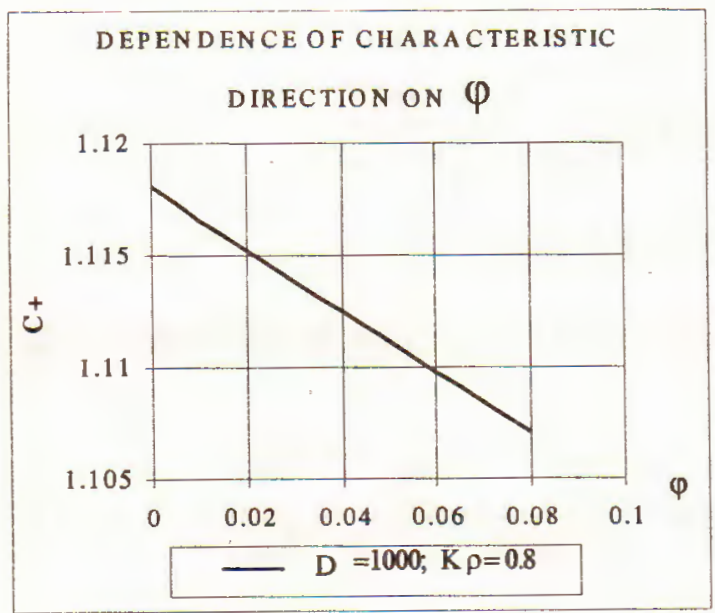

Fig. 3

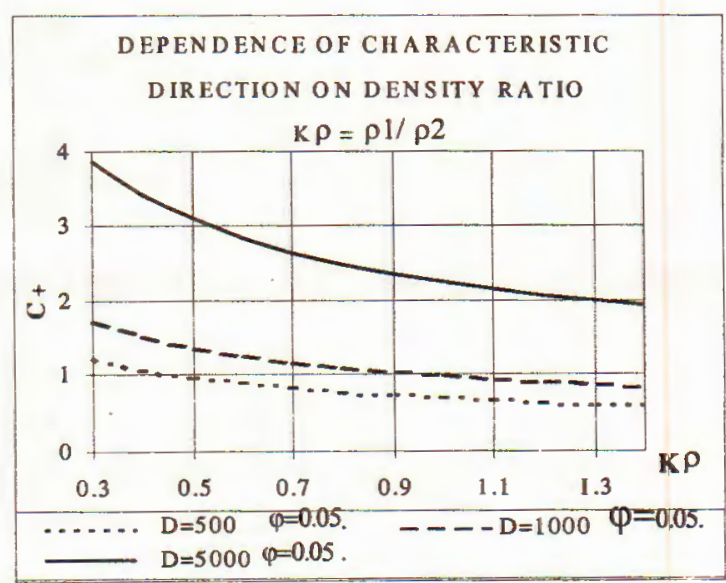

Fig. 2

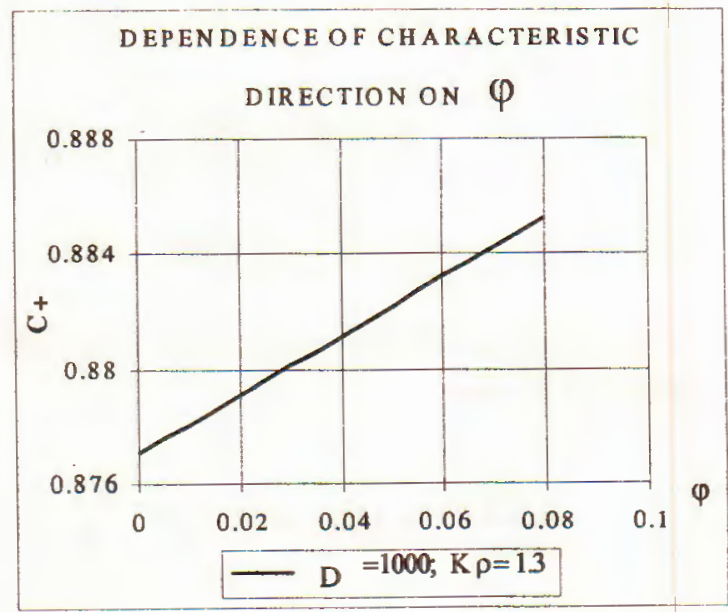

Fig. 4

\subsection{The characteristic form of governing equation system}

To receive the characteristic form of the governing equation system, first of all, 
let us to rewrite it into vector form $[4,5]$ :

$$
\frac{\partial \bar{W}}{\partial t}+\bar{M} \frac{\partial \bar{W}}{\partial z}=\bar{B}
$$

where column vectors $W$ and $B$, matrix of coefficients $M$ is

$$
\bar{W}=\left(\begin{array}{l}
\varphi \\
J
\end{array}\right) ; \quad \bar{M}=\left(\begin{array}{cc}
0 & a_{1} \\
a_{2} & 0
\end{array}\right) ; \quad \bar{B}=\left(\begin{array}{l}
0 \\
f
\end{array}\right) .
$$

Let's decompose the matrix $\bar{M}$ into the product of second order matrix $\bar{P}$ as:

$$
\bar{M}=\bar{P}^{-1} \bar{D} \bar{P}
$$

where $\bar{D}$ is the diagonal matrix of eigenvalues of the system (2.5):

$$
\bar{D}=\left(\begin{array}{cc}
\lambda_{1} & 0 \\
0 & \lambda_{2}
\end{array}\right)
$$

Taking into account (2.8), the equation (2.7) yields

$$
\bar{P} \frac{\partial \bar{W}}{\partial t}+\bar{D} \bar{P} \frac{\partial \bar{W}}{\partial z}=\bar{P} \bar{B}
$$

Using the notations $\bar{P}_{i}$ as $i$-row vector of $\bar{P}$, equation $(2.10)$ can be rewritten in the form:

$$
\bar{P}_{i}\left(\frac{\partial \bar{W}}{\partial t}+\lambda_{i} \frac{\partial \bar{W}}{\partial z}\right)=\bar{P}_{i} \bar{B}
$$

Now, consider the variation of $d \bar{W}$ on the characteristic curves $C_{1,2}$, corresponding to $\lambda_{1,2}$ in the plane of coordinates $(t, z)$ :

$$
\left.d \bar{W}\right|_{C_{i}}=\left.\left(\frac{d \bar{W}}{d t} d t+\frac{d \bar{W}}{d z} d z\right)\right|_{C_{i}}=\left.d t\left(\frac{d \bar{W}}{d t}+\lambda_{i} \frac{d \bar{W}}{d z}\right)\right|_{C_{i}}
$$

With (2.12), equation (2.11) along the characteristic curves $C_{1,2}$, has the form:

$$
\left.\bar{P}_{i}\left(\frac{d \bar{W}}{d t}\right)\right|_{C_{i}}=\left.\bar{P}_{i} \bar{B}\right|_{C_{i}}
$$

or

$$
\begin{aligned}
& \left(\frac{d \varphi}{d t}\right)_{C_{1}}+\sqrt{\frac{a_{1}}{a_{2}}}\left(\frac{d J}{d t}\right)_{C_{1}}=\left.\sqrt{\frac{a_{1}}{a_{2}}} f\right|_{C_{1}} \\
& \left(\frac{d \varphi}{d t}\right)_{C_{2}}-\sqrt{\frac{a_{1}}{a_{2}}}\left(\frac{d J}{d t}\right)_{C_{2}}=-\sqrt{\left.\frac{a_{1}}{a_{2}} f\right|_{C_{2}}}
\end{aligned}
$$


The equation system (2.13) or (2.14) are a characteristic form of governing equations. It is not difficult to show that the characteristic equations (2.14) can be rewritten in the folowing form: (16) where:

$$
\begin{aligned}
& \left.\frac{d}{d t}\left(a_{3} y^{1 / 2}+J\right)\right|_{C_{1}}=\left.f\right|_{C_{1}} \\
& \left.\frac{d}{d t}\left(a_{3} y^{1 / 2}-J\right)\right|_{C_{2}}=-\left.f\right|_{C_{2}}
\end{aligned}
$$

where

$$
\begin{aligned}
a_{3} & =\frac{2 \sqrt{\rho_{1} D K_{\rho}}}{\left(1-K_{\rho}\right)} ; \quad y=K_{\rho}+\varphi\left(1-K_{\rho}\right) \\
f & =K_{2} K_{\rho} J \frac{(1-y)\left(y-K_{\rho}\right)}{y\left(1-K_{\rho}\right)^{2}}+K_{3} K_{\rho} \frac{(1-y)\left(y-K_{\rho}\right)}{y\left(1-K_{\rho}\right)^{2}}
\end{aligned}
$$

\section{The solution for stationary sedimentation}

In a stationary case the particle sedimentation is described by one unique equation:

$$
\frac{d \varphi}{d z}=-\frac{g\left(\rho_{1}-\rho_{2}\right)}{D} \varphi(1-\varphi)
$$

and the solution $\varphi$ has the form:

$$
\varphi=\frac{1}{1+A e^{\alpha x}}
$$

where $\alpha=\frac{g\left(\rho_{1}-\rho_{2}\right)}{D}$.

To determine the constant $A$, it has to use the conservation condition of particles phase in the considering space:

$$
\frac{1}{L} \int_{0}^{L} \varphi d z=\varphi_{0}=\text { const. }
$$

From (3.2) and (3.3) we will have

$$
\varphi=\frac{1}{1+\left[\frac{e^{\left(1-\varphi_{0}\right) \alpha L}-1}{e^{\alpha L}-e^{\left(1-\varphi_{0}\right) \alpha L}}\right] e^{\alpha z}}
$$

where $\quad \alpha=\frac{g\left(\rho_{1}-\rho_{2}\right)}{D}$. 
The dependence of volume-concentration $\varphi$ on density ratio $\rho_{1} / \rho_{2}$ and diffusion coefficient $D$ is demonstrated in Figs. 5, 6 and 7.

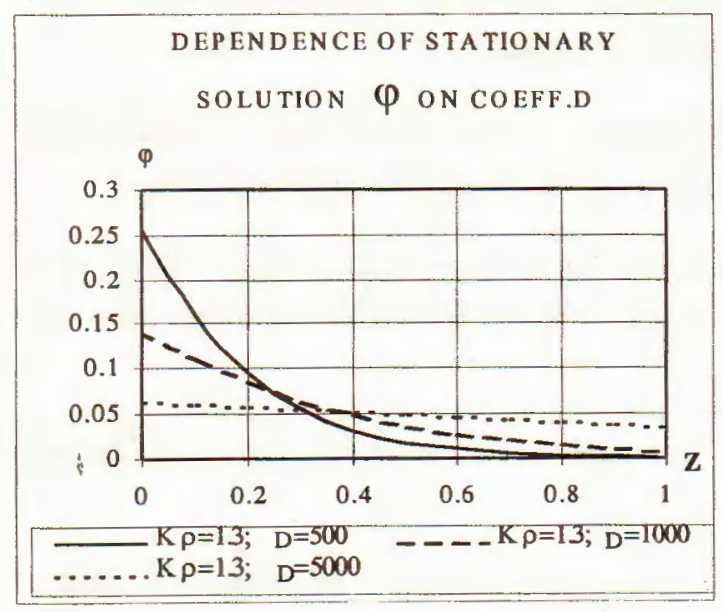

Fig. 5

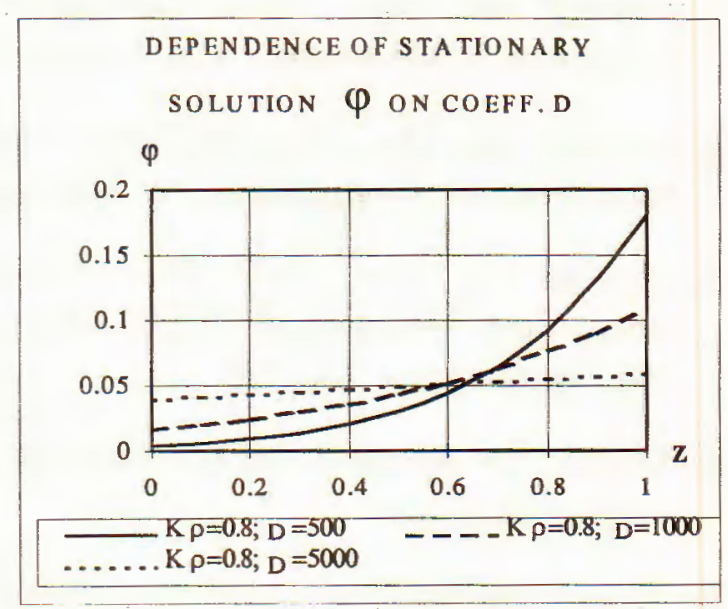

Fig. 6

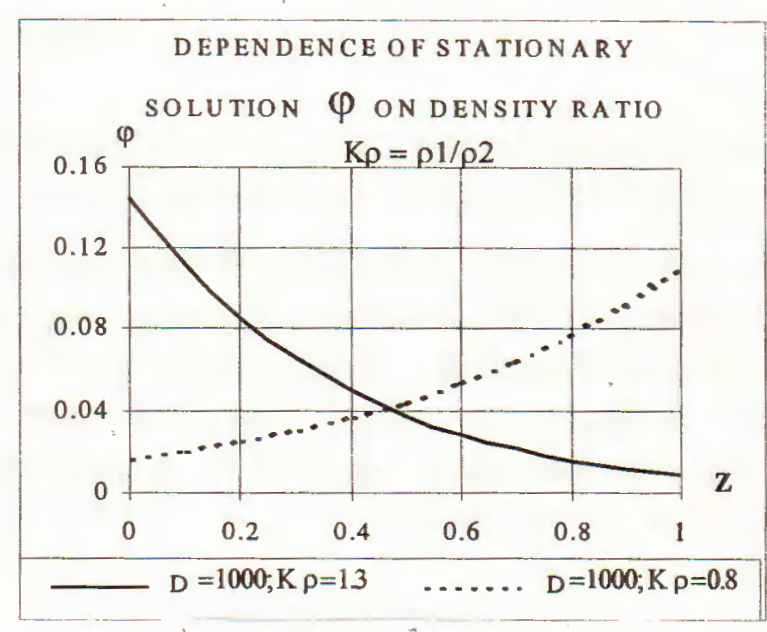

Fig. 7

\section{Conclusion}

It has been shown some preliminary results in application of the generalized diffusion theory on modelling the sedimentation process of rigid particles in stationary viscous suspension. Obviously, the obtained results are exceptionally qualitative, because many phenomenological parameters and coefficients have to be determined from the experiments.

Acknowledgement. This work was financial supported by the Vietnamese National Foundation for Basic Research in Natural Sciences. The authors would like to thank Dr. Dinh Van Manh, Dr. Hoang Van Lai for their useful discussion. 


\section{REFERENCES}

1. Nguyen Van Diep. To the generalized diffusion theory of mixtures, Intern. J. Advances in Mechanics, V. 1 1, N.2, 1988 (in Russian).

2. Nguyen Van Diep, Nguyen Hong Phan. Theory of two-phase flow of fluid with rigid ellipsoidal particles, J. of Mechanics, V. 15, No. 3,1993 (in English).

3. Nguyen Van Diep. Hydrodynamical modeling of deformable particle-fluid twophase flow, Proceeding of 2nd Intern. Conference on Multiphase Flow 95- Kyoto,Japan, April 3-7,1995.

4. Michael B. Abbott. An introduction to the method of characteristics, Thames and Hudson 1966.

5. Mecanique des fluides appliques, Pierre-Lous Violllet, Jean Paul Chabard, Pascal Esposito, Diminique Laurence, Ecole nationale de ponts et chaussces, 1997-1998.

Received june 25, 2001

\section{LÝ THUYẾT KHUYÊCH TÁN SUY RỘNG CHO SƯ LẮNG ĐỌNG CỦA HAT CẦU RẮN KHÔNG QUAY TRONG CHẤT LỎNG NHỚT}

Bài báo trình bày một áp dụng của Lý thuyết khuyếch tán suy rộng cho bài toán lắng đọng của các hạt cầu rắn, không quay được trong chất lỏng nhớt. Các phương trình cơ bản đã được thu nhận. Trong trường hợp này, các tác giả dã chỉ ra rằng hệ phương trình có dạng hypecbolic và đưa ra các phương trình trên đường dặc trưng. Các tính chất toán học của hệ phương trình cũng như lời giải dừng cho bài toán lắng đọng đã được nghiên cứu số. 